International Journal of Information Technology Convergence and Services (IJITCS) Vol.2, No.2, April 2012

\title{
IMPACT-Intelligent Memory Pool Assisted Cognition Tool : A Cueing device for the Memory Impaired
}

\author{
S.Cyril Naves \\ Department of Information Technology,Sri Sairam Institute of Technology, Anna \\ University, India
}

cyrilnaves91@gmail.com

\begin{abstract}
:
Memory impairment results from a variety of disease such as Alzheimer, Parkinson, brain trauma and Aging. These people are obsessed with their current state and emotions when dealing with their environment with deteriorating memory in them. There are about 30 million people worldwide afflicted with this instance. They lose their insight into their own condition, forgetting even their loved ones, disability to locate their residence and a myriad of other inabilities. Medicine has evolved fast enough but still there is not a definite method to diagnose or a treatment for their memory loss. The only finding has revealed is due to the depletion of the brain cells and loss in hormone secretion to carry sensory messages. The appropriate reason for this behavior of cells is a mystery unsolved. While not only losing their pensive they develop a cold behavior, socially and emotionally they become unbalanced. The most common form of assistance to them is by caregivers or life logging through writing their reminiscences, taking photos of their surrounding and their close people, mobile phones etc. While all these can be of some form of help to this people it will result only in more stress to their already damaged brain resulting in depression and other mental problems. The proposed tool IMPACT acts like a second brain with a repository of memory comprising of their previous experiences in multimedia format which is sensed and fetched when they come across a similar person or surrounding thus enabling them to recall their senses about that situation engaging cueing interaction.
\end{abstract}

\section{Keywords:}

image classification, feature extraction, segmentation, Fuzzy logic, audio recognition, cognition.

\section{Introduction}

The brain is the most superior organ a human is gifted with enabling him to acquire data, consolidate and retrieve it. The major challenges a person comes across when his brain cells deteriorate causing damage to his cognition capabilities. Many people around the world are diagnosed with memory loss and this disability resulting as a side effect of many diseases is expected to increase many fold.[2] This disease makes a person to lose his current and depress him to sole reliance upon others forgetting their activities, family members and even their 
International Journal of Information Technology Convergence and Services (IJITCS) Vol.2, No.2, April 2012 purpose in life.IMPACT is the device designed to trigger the cue of a person enabling him to recall his sense enabling him to know about his past, and direct his present. This device gives the person anew lease of life to lead an independent, active and a stress free life.

\section{Background}

One out of every hundred people around the world over the age of 50 have been diagnosed with dementia resulting as a side cause of trauma, aging Alzheimer etc.[1] Memory impairment can lead to feelings of uncertainty, irritation, frustration and fear in the person. Living with constant uncertainty about previous experiences can lead to a frightening loss of control in people's lives. [3] Increasingly dependent on a caregiver to help make simple decisions, they are forced to relinquish their life. They can become easily depressed as they struggle with failures with the loss of control that pervades their life. By creating a pool of their memories with sensing their encounter through audio and video format and then offering a quick glimpse into their previous reflection with that situation allow people to reflect on their life and perceive themselves living and experiencing reality continuously through time.

\section{Component Specifications}

Image Sensor: The image sensor built in is a CMOS sensor which is a type of active pixel sensor. This converts the incoming light into voltage and the transfers to a memory. The technical specification of the sensor suitable will be of

Capturing speed: 50frame per second

Width: 1600

Height: 1200

Aspect ratio: $4: 3$

Actual pixel count: $1,920,000$

Mega pixel: 4

Night vision: Bright white light led.

3.1 Processor: Cortex-A8 processor is used with enabled NEON technology based packed SIMD processing. Registers are considered as vectors of elements of same type. The processor is enabled for accelerating multimedia application. Its frequency ranges from $600 \mathrm{MHz}$ to $1 \mathrm{GHz}$ with a superscalar micro architecture.

3.2 Memory: A ROM memory is used with a faster access mode for a cache for it. "Smart" memory card architecture is used with significantly increased performance by a fast dynamic random access memory which allows up to 8byte data transfers after every $27 \mathrm{~ns}$ after initial access.

3.3 Power: A rechargeable Lithium-ion button cell is used with relatively $1000 \mathrm{mAh}$ is implemented.

3.4 Speaker: A micro speaker is fit in so as to present the user with the recorded sound after comparison of the present sound wave with the previous alias one. 
3.5 Microphone: It is built in the power range of $5 \mathrm{v}$ so as to capture analog sound and the send it for preprocessing before comparing for an alias sound wave pattern and presenting via speaker giving a glimpse of recording.

Sample Rate: $150 \mathrm{kHz}$

Bit Rate: 24bit

Polar Patterns: Cardioid, Bidirectional, Omni-directional \& Stereo

Frequency Response: $20 \mathrm{~Hz}-20 \mathrm{kHz}$

Sensitivity: $4.5 \mathrm{mV} / \mathrm{Pa}(1 \mathrm{kHz})$

Max SPL: $80 \mathrm{~dB}$

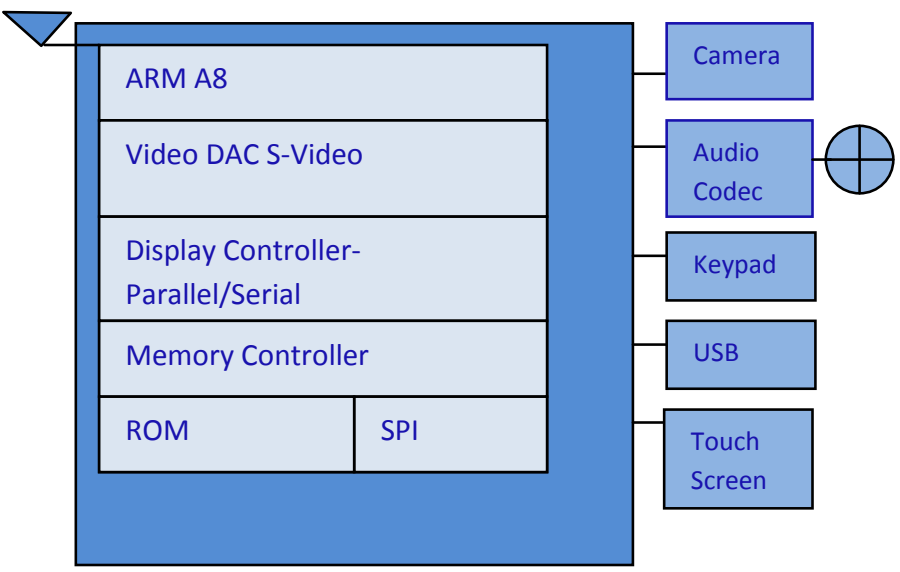

Fig 1 Block diagram of IMPACT

\section{Architecture}

IMPACT is enabled with an audio and video sensor with live capturing mode so as to record a particular experience of a person directed towards a central memory system in it.[2] It then fetches the already stored comparing with the recently recorded memory by feature matching of either audio or video pattern.

[3]The intelligent memory pool works in a manner to avoid memory clogging causing low access speed. Each and every audio and video is first feature extracted an then stored in the database It stores in only the latest memory associated with that particular event or person and the existing earlier encounters are automatically deleted thereby avoiding large memory space and fetching delay. 


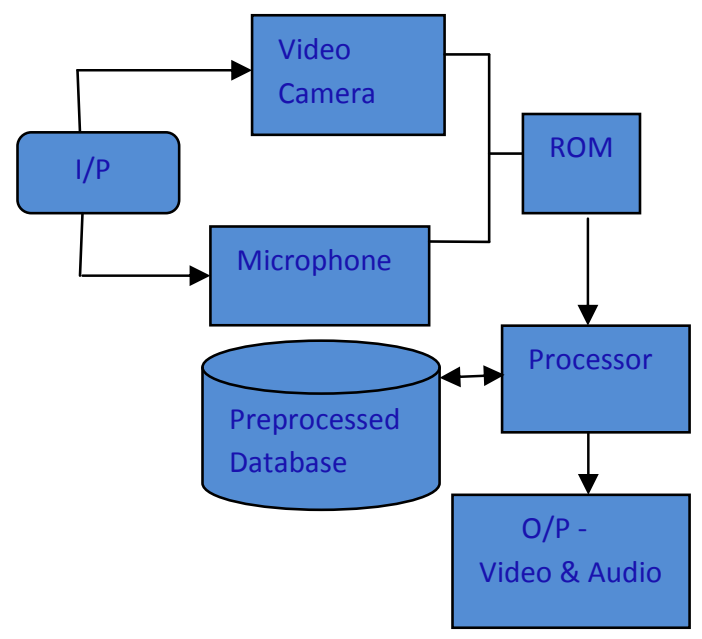

Fig 2.Working Layout of IMPACT

\section{Implementation and Working:}

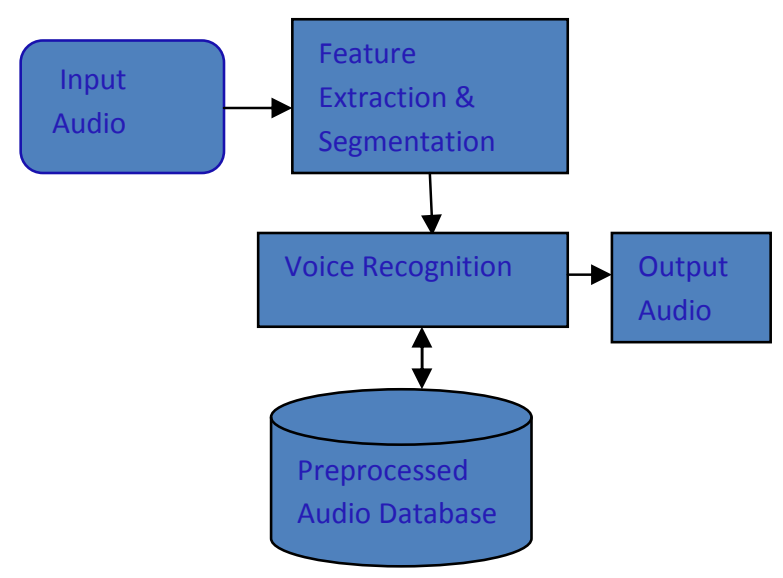

Fig 3.Functional Block Diagram of Audio Recognition

5.1 Audio Recognition: The audio being recorded with the surrounding of the person i.e. a query audio is processed and segmented and then compared with the already existing featured audio database. After feature matching it is then played to the user allowing him to trigger his cognition. Then the feature extracted query audio is stored in the memory deleting the previous edition of the event. 


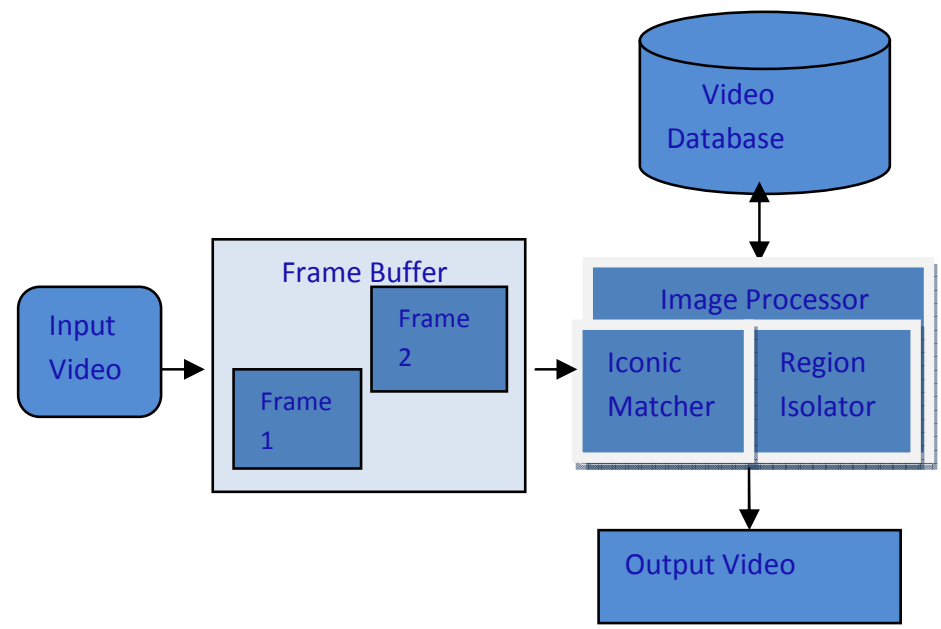

Fig4. .Functional Block Diagram of Video Recognition

5.2 Video recognition: The image from the video camera is passed on to a frame buffer following the frame is isolated into regions for each object or regions. [6]Then a feature analyzer computes asset of global and local features for each region and based on those features selects several reference patterns forming an associated attribute memory. This attribute memory is compared and the relative video set is played to the user recollecting his past. The featured video is stored in the memory deleting the earlier one. The user interface is designed with several modes to facilitate the person for options with deletion, storing or fetching memory mode with it.

\section{Algorithm deployed in IMPACT:}

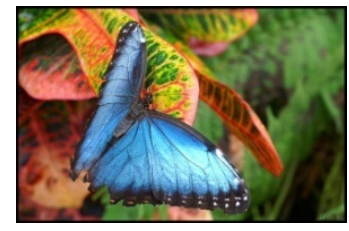

Fig 5.Input Video frame

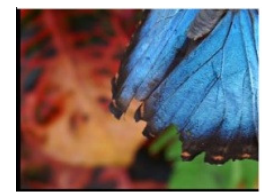

Fig 6.Region Isolated frame 1 


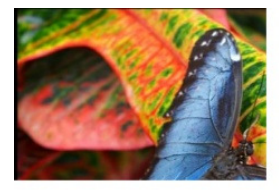

Fig 7.Region Isolated frame 2

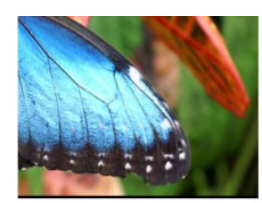

Fig 8.Region Isolated frame 3

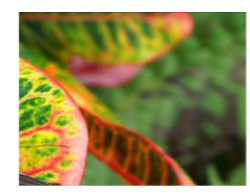

Fig 9.Region Isolated frame 4

\subsection{Video recognition:}

6.1.1 Preprocessing: In the video recognition a fuzzy logic approach of unified feature matching is done for region based image retrieval.[4] In this each frame is represented by a set of segmented region each of which is characterized by a fuzzy feature reflecting color, texture and shape properties. As a result each frame is associated with a family of fuzzy feature corresponding to regions. Fuzzy features naturally characterize the gradual transition between regions within an image and incorporate the segmentation related uncertainties into the recognition algorithm.

6.1.2 Iconic matcher: The resemblance of two images is then defined as the overall similarity between two families of fuzzy features and quantified by an iconic matcher, which integrates properties of all the regions in the images. [4]Compared with similarity measures based on individual regions and on all regions with crisp-valued feature representations, the UFM measure greatly reduces the influence of inaccurate segmentation and provides a very intuitive quantification. 


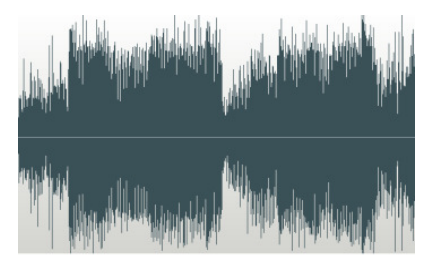

Fig 10.Cepstral analyzed Query sound wave

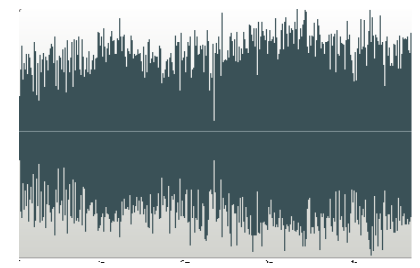

Fig 11.Cepstral analyzed sound database

\subsection{Audio recognition:}

6.2.1 Processing: The recorded incoming audio is first processed by neural networks in a way analyzing both text dependent and text independent sound avoiding external disturbances .[5]Each sound wave is extracted of its feature data including cepstral analysis of it.

2) Feature Matching: Each analyzed sound wave is compared with the already existing feature extracted database, and then if the feature corresponds then the previous audio wave cepstral analysis and the current ones are matched to get the highest occurrence of event.

7. Conclusion: This system IMPACT will allow the person with memory impairment to review a multimedia narrative of an experience as cues to help them not only to recall the experience but to be able to relive the experience. Instead of repetitively asking for assistance, they will be able to use this device as a tablet in recollecting their experience.

8. Future Work: This system has to be deployed with memory impaired individuals and their peers. The effectiveness of the device has to be evaluated and also more concentration has to be dealt in the user interface. Based on caregiver's authored content enhancement of the richness of the review experience has to be done.

\section{References:}

[1] Alzheimer's Association. Families Care: Alzheimer's Care giving in the United States 2004.

[2] Kawamura, T., Kono, Y. and Kidode, M. Wearable interfaces for a video diary: towards memory retrieval, exchange, and transportation ISWC 2002, 31- 38.

[3] X S Zhou and T S Huang. Relevance feedback in image retrieval: A comprehensive review. Multimedia Systems, 8:536-544, 2003. 
International Journal of Information Technology Convergence and Services (IJITCS) Vol.2, No.2, April 2012

[4] J Zobel and T Hoad. Detection of video sequences using Compact signatures. ACM Trans. Inf. Syst., 24(1):1-50, 2006.

[5] A. L. Higgins, L. Bahler, and J. Porter, "Speaker verification using randomized phrase prompting", Digital Signal Processing, Vol. 1, 1991, pp. 89-106.

[6] P. Melin, M. L. Acosta, and C. Felix, "Pattern Recognition Using Fuzzy Logic and Neural Networks", ProceedingsofIC-AI'03,LasVegas,USA,2003,pp.221-227. 